\title{
Aluminum toxicity and behavior in the weanling Long-Evans rat
}

\author{
B. MICHAEL THORNE, ART COOK, TIM DONOHOE, STEVE LYON, \\ DENIS M. MEDEIROS, and CHRIS MOUTZOUKIS \\ Mississippi State University, Mississippi State, Mississippi
}

\begin{abstract}
Muricidal behavior, weight, open-field activity, passive-avoidance learning, and learning of an eight-arm radial maze were assessed in Long-Evans hooded rats subjected as weanlings to 60 days of oral administration of aluminum hydroxide gel (Group AA) or tap water (Group NA). Measurement of brain aluminum content indicated that the groups did not differ significantly in neural incorporation of aluminum, although the median amount of aluminum in each brain area examined was higher for Group AA than for Group NA. Although the highest brain concentrations of aluminum were found in the hippocampus, there was no evidence for cognitive impairment in the present study. Aluminumexposed rats weighed less than nonexposed animals at all weight periods, probably reflecting their initially decreased fluid intake. There was slight evidence for activity changes as a function of brain aluminum content. Age of the animals appears to be an important consideration in the study of aluminum toxicity in rats, with young animals less affected than older animals.
\end{abstract}

Aluminum toxicity has been suggested as a cause for at least some cases of Alzheimer's disease (see, e.g., Crapper, Krishnan, \& Dalton, 1973; Crapper, Krishnan, \& Quittkat, 1976). In addition, research with animals has often found evidence for behavioral impairment following exposure to aluminum. For example, cats injected intracranially with aluminum chloride were impaired on both short-term retention and a conditioned avoidance response (Crapper \& Dalton, 1973). Rabbits injected intraventricularly with aluminum tartrate showed deficits on both the learning and retention of a step-down active avoidance task (Petit, Biederman, \& McMullen, 1980).

Studies in which aluminum compounds have been administered to rats have produced inconsistent results. For example, aluminum-injected rats did not show neurofibrillary degeneration or the progressive encephalopathy seen in cats, even at brain concentrations several times those used in cats (King, DeBoni, \& Crapper, 1975). King et al. did report significant correlations between brain aluminum content (BAC) and some performance measures, however, with high aluminum levels being associated with poorer performance on a conditioned avoidance response.

By contrast, in a study by Bowdler et al. (1979), aluminum was absorbed and deposited in the brains of rats to which it was administered by oral intubation. High neural levels were associated with increased activity, decreased roto-rod performance, and increased sensitivity to flicker. In another study, aluminum administered

The research was in part supported by an institutional grant from the National Science Foundation to Mississippi State University. The authors would like to thank Rosemary Wander for her assistance in preparing the brain samples for analysis. Denis M. Medeiros's present address is: Division of Home Economics, Box 3354, University Station, Laramie, WY 82071. Send reprint requests to B. Michael Thorne, Drawer 6161, Mississippi State, MS 39762. to rats in an aqueous suspension of Amphojel (an antacid containing aluminum hydroxide) resulted in poor performance on an eight-arm radial maze (Hinz \& Dufort, 1983). Unfortunately, Hinz and Dufort did not analyze BAC and were unable to attribute the observed memory deficit to the effects of the neural incorporation of aluminum.

In a recent study from our laboratory (Thorne et al., 1986), adult rats were fed ground rat chow containing one of four different levels of added aluminum hydroxide. After 30 days on the diets, the animals were tested on a variety of measures, including mouse killing, open-field activity, passive-avoidance (PA) learning, and a discrimination task with reversal training. High levels of aluminum were correlated with decreased open-field activity, deficient PA performance, and increased errors on the discrimination task with reversal. The highest levels of aluminum were found in the hippocampus.

The purpose of the present study was to determine the effect of a more lengthy period of aluminum administration on initially younger rats, since it is probable that the age of the animal is a factor in aluminum toxicity (Commissaris et al., 1982; Petit, Biederman, Jonas, \& LeBoutillier, 1985; Wisniewski, Sturman, \& Shek, 1980). Specifically, weanling Long-Evans rats were exposed to different levels of aluminum hydroxide gel for 60 days prior to behavioral testing. Testing consisted of measurements of open-field activity, muricide, PA learning, and eight-arm radial maze learning. BAC assays were performed after testing.

\section{METHOD}

\section{Subjects}

The subjects were 16 Long-Evans male hooded rat weanlings taken from the animal colony maintained by the psychology department at Mississippi State University. All animals were approximately 22 days old 
at the beginning of the study. Because of the premature death of 1 rat in the aluminum group, data are reported on 15 subjects.

The animals were randomly assigned to one of two groups: no added aluminum (Group NA, $n=8$ ) and added aluminum (Group AA, $n=7$ ). Animals in Group NA received tap water with 1 drop of peppermint extract (Kroger Co., Cincinnati, $\mathrm{OH}$ ) added per $100 \mathrm{ml}$ water in an attempt to equate taste for the two groups. Rats in Group AA received tap water plus a peppermint-flavored liquid antacid containing aluminum hydroxide (Rugby Laboratories, Inc., Rockville Centre, NY) mixed in a concentration of $80 \mathrm{ml}$ water and $20 \mathrm{ml}$ antacid. The amount of aluminum hydroxide in $20 \mathrm{ml}$ of the antacid was $1,280 \mathrm{mg}$, and the average daily dosage for Group AA over the 60-day administration period was approximately $2,400 \mathrm{mg} / \mathrm{kg}$.

\section{Apparatus}

Two apparatuses were used to assess open-field activity, one measuring $42 \times 42 \times 15 \mathrm{~cm}$ and the other measuring $76.2 \times 76.2 \times 25.4 \mathrm{~cm}$. The smaller box was painted flat white and had black lines dividing the floor into 25 equal squares. The larger apparatus was painted flat black and had white lines dividing the floor into 25 equal squares. Both boxes were covered with wire-mesh tops.

The PA apparatus consisted of two chambers: an anterior chamber measuring $24.7 \times 12.7 \times 30.7 \mathrm{~cm}$ constructed of plywood with a wooden floor, and a posterior chamber measuring $30.5 \times 26.7 \times 29.0 \mathrm{~cm}$ constructed of plywood with a grid floor. Both chambers were painted flat black. The chambers were separated by a wooden partition extending the width of the apparatus. A 15 -W bulb was mounted in the anterior chamber to provide light for the test and to increase the discriminability of the chambers.

The eight-arm radial maze was constructed from plywood painted flat black and was modeled after the one used by Olton and Samuelson (1976). The maze was supported by wooden legs and stood $60 \mathrm{~cm}$ above the floor.

\section{Procedure}

On the day of weaning (i.e., at approximately 22 days of age), all animals were weighed, tested for open-field activity, and assigned randomly to the different treatments. The weight of each rat was recorded on a weekly basis for 10 weeks, and fluid intake was measured daily by subtracting the fluid remaining in a rat's bottle from $100 \mathrm{ml}$ (the amount given to each subject daily).

Open-field activity was assessed on a biweekly basis. For the openfield test, each animal was placed singly onto the center square of the apparatus, and the number of squares crossed with the hind legs and the number of rearings (standing on the hind legs) were recorded during a 1-min period. The smaller apparatus was used for the first two measurement periods, and the larger was used for the last three assessments. Frequent interobserver reliability sessions were performed with the use of an independent observer. The reliability of horizontal activity scores was $r=.97(p<.01)$, and that for vertical activity was $r=.98$ $(p<.01)$. The apparatus was cleaned between subjects, and the order of testing was varied from week to week.

On the day after termination of the aluminum administration, all rats were tested for muricidal behavior. For the test, an albino mouse was placed into the cage of each rat, and the incidence of killing and latency to kill were observed for $20 \mathrm{~min}$. After $24 \mathrm{~h}$, any live mice or the remains of dead ones were removed from the cage. Any kills taking place after the 20-min observation period were assigned a latency of $24 \mathrm{~h}$.

Training on the PA task began on the day following completion of the mouse-killing test. Training consisted of placing each rat into the lighted anterior compartment. After a 3-sec period, the door was raised to allow the rat to enter the unlighted posterior chamber, and latency to leave the lighted chamber was recorded. Entry into the darkened compartment resulted in the lowering of the guillotine door and the application of a 3-sec, 1-mA footshock. The subject was then removed from the apparatus and returned to its cage to await retention testing.

Twenty-four hours after training, each rat was placed into the anterior chamber, the guillotine door opened, and the latency to enter the darkened chamber recorded. The retention test was terminated either upon the animal's entry into the posterior compartment or when the animal had remained in the lighted chamber for $3 \mathrm{~min}$.
On the day after completion of PA training, all animals were weighed and placed on a food-deprivation schedule designed to reduce their weight by approximately $15 \%$. When this criterion was reached, 3 days of familiarization with the eight-arm radial maze began. On Day 1 , the animals were placed in pairs on the maze for $10 \mathrm{~min}$ with $45-\mathrm{mg}$ Noyes pellets scattered randomly throughout. On Days 2 and 3 , the rats were placed individually on the maze for $5 \mathrm{~min}$ each; Noyes pellets were scattered throughout the maze on Day 2 but were restricted to the vicinity of the reward wells on Day 3.

Beginning on Day 4, each rat received one training trial per day on the maze. A trial consisted of placing the animal in the center of the maze and removing it either after it had been reinforced on each of the eight arms or after $10 \mathrm{~min}$ had elapsed. Reinforcement consisted of one 45-mg Noyes pellet placed in the reward hole at the end of each arm. Correct choices and errors were recorded on each trial, an error being defined as choice of a previously chosen arm on which the pellet had been consumed. Training continued until each animal reached a criterion of seven out of eight correct choices for 5 consecutive days or until 20 days had elapsed. The apparatus was cleaned between the testing of the animals.

Following completion of the maze task, all animals were weighed and sacrificed with an overdose of sodium pentobarbital. To control for a possible decrease in BAC with the passage of time following termination of exposure, we sacrificed all subjects at the same time. Brains were quickly extracted and dissected into three portions: the neocortex dorsal to the rhinal fissure, the hippocampus, and the rest of the brain excluding the olfactory bulbs. Brain sections were then weighed, placed in acid-washed Nalgene test tubes, and dried in a vacuum oven for at least $72 \mathrm{~h}$ at $38^{\circ} \mathrm{C}, 63.5 \mathrm{~mm} \mathrm{Hg}$. Tissues were reweighed after drying to obtain a dry weight and shipped to the University of Wyoming for aluminum analysis.

Tissues were transferred to $50-\mathrm{ml}$ acid-washed glass beakers. Watch glasses were placed on the beakers during digestion and ashing. Brain parts were digested in $10 \mathrm{ml}$ of nitric acid prepared for trace-element analysis (Fisher Scientific Co., Fairlawn, NY) and wet-ashed in a solution containing $10 \mathrm{ml}$ of nitric acid and $2 \mathrm{ml}$ of $70 \%$ perchloric acid prepared for trace-element analysis until white fumes evolved. The remaining residue from the neocortex and rest-of-brain samples was transferred to 10-ml volumetric flasks and diluted up to volume with distilleddeionized water and rinsings from the beakers. The hippocampal residue was treated in a similar manner, except that it was transferred to 5-ml volumetric flasks. Several blank digests were run simultaneously with other samples to correct for any background contamination from the acids and glassware.

Aluminum content was analyzed by an inductively coupled argon plasma spectrophotometer (Perkin-Elmer Model No. 5500, Norwalk, CT) at a wavelength of $396.15 \mathrm{~nm}$ and a slit setting of $0.02 \mathrm{~nm}$ on low. An integration time of $0.2 \mathrm{sec}$ with a 30-sec delay was used. Samples were read in triplicate and averaged. Aluminum standards in the linear working range were prepared from Certified Atomic Absorption Standards (Fisher Scientific Co.). BAC was expressed as a function of both wet and dry weights.

\section{RESULTS}

\section{Brain Aluminum Content}

Because comparable results were obtained when the data were expressed either as wet weights or as dry weights, only the wet weights are reported. There were no significant differences between Group NA and Group AA in BAC for any brain part analyzed. However, the group medians were in the appropriate direction, with Group AA having the larger median for each brain area (all values are expressed in parts per million [ppm]); neocortexGroup NA $=5.46$, Group AA $=7.60$; hippocampusGroup NA $=24.29$, Group AA $=28.08$; rest-ofbrain-Group NA $=3.16$, Group $\mathrm{AA}=4.26$; whole brain-Group NA $=4.77$, Group $A A=8.20$. 
Table 1

Average Body Weight (in Grams) and Standard Errors by Week of Exposure

\begin{tabular}{lcccccccccc}
\hline & \multicolumn{10}{c}{ Week } \\
\cline { 2 - 10 } Group & 0 & 1 & 2 & 3 & 4 & 5 & 6 & 7 & 8 \\
NA & $44.6 \pm 3.0$ & $78.4 \pm 3.8$ & $128.4 \pm 6.0$ & $177.0 \pm 4.7$ & $230.4 \pm 4.7$ & $275.4 \pm 3.9$ & $314.9 \pm 4.0$ & $349.5 \pm 4.0$ & $369.6 \pm 3.3$ & $394.5 \pm 2.9$ \\
AA & $42.3 \pm 2.7$ & $61.0 \pm 5.7$ & $102.7 \pm 8.1$ & $141.4 \pm 10.3$ & $187.9 \pm 12.1$ & $224.6 \pm 16.0$ & $249.7 \pm 25.6$ & $294.1 \pm 11.7$ & $328.9 \pm 17.6$ & $356.9 \pm 17.2$ \\
$F(p)$ & & $6.7(.023)$ & $6.7(.023)$ & $10.8(.006)$ & $11.8(.004)$ & $10.8(.006)$ & $7.3(.018)$ & $22.3(.000)$ & $5.9(.030)$ & $5.3(.038)$ \\
\hline
\end{tabular}

Note-Week 0 was the time of weaning. $F$ values and probabilities are shown for each significant comparison.

A comparison of aluminum incorporation across brain parts revealed evidence for significant between-part differences $(H=24.74, p<.01)$. All pairwise comparisons were significant, with hippocampal samples having more aluminum than either neocortical or rest-of-brain samples $(p<.001)$ and neocortical samples having more aluminum than rest-of-brain samples $(p<.02)$. The brain part medians (in ppm) were: hippocampus, 25.86; neocortex, 7.57; rest-of-brain, 3.97 .

\section{Body Weight and Fluid Intake}

A two-way ANOVA performed on the body weights by groups over time revealed that there was a significant groups effect $(F=12.83, p<.01)$, a significant effect over weeks $(F=469.91, p<.001)$, and a significant interaction $(F=2.63, p<.02)$. Body weight in Group NA was significantly greater than that in Group AA at each week of observation after exposure to the aluminum-hydroxide gel. Mean weights, standard errors, $\boldsymbol{F}$ values, and probabilities across weeks are given in Table 1. The significant effect over weeks indicates that the rats gained weight throughout the study, whereas the significant interaction reflects the fact that Group NA animals gained weight more rapidly than did Group AA rats.

Because of the weight differences between groups, fluid intake was converted to $\mathrm{ml} / 100 \mathrm{~g}$ body weight. A twoway ANOVA on fluid intake as a function of body weight by groups over time revealed that the main effect for groups was not significant; there was a significant decrease in intake over time $(F=34.85, p<.001)$; and there was a significant interaction. Further analysis of the interaction revealed that Group AA rats had a lower fluid intake on the first week of exposure $(F=4.8, p=.047)$. The converted daily averages and standard errors are shown in Table 2.

Spearman correlation coefficients revealed that rest-ofbrain aluminum content was significantly related to body weight at 6 of the 10 measurement periods $(p<.05$ in each case): Week $2, r_{\mathrm{S}}=-.45$; Week $3, r_{\mathrm{S}}=-.47$; Week 4, $r_{\mathrm{s}}=-.46$; Week 6, $r_{\mathrm{s}}=-.50$; Week 7, $r_{\mathrm{S}}=-.49 ;$ Week 8, $r_{\mathrm{S}}=-.50$. The aluminum content of other assayed brain areas was not related to body weight. BAC was not related to fluid intake.

\section{Muricide}

The groups did not differ in mouse-killing behavior; none of the Group NA rats killed and only 1 of the Group AA rats exhibited the response.

\section{Open-Field Activity-Horizontal and Vertical}

There were no between-groups differences at any of the measurement periods for either horizontal activity or rearing in the open field. There were three significant positive correlations between BAC and horizontal activity: neocortical aluminum was related at the fourth measurement period $\left(r_{\mathrm{s}}=.48, p<.04\right)$; whole brain aluminum was related at the fourth period $\left(r_{\mathrm{s}}=.46, p<.05\right)$; rest-ofbrain aluminum was related at the fifth period $\left(r_{\mathrm{s}}=.45\right.$, $p<.05)$. There were three significant negative correlations between BAC and vertical activity: rest-of-brain aluminum was correlated at both the first and third periods ( $r_{\mathrm{s}}=-.44$ and $-.80, p<.05$ and .01 , respectively); whole brain aluminum was correlated at the third period $\left(r_{\mathrm{S}}=-.64, p<.01\right)$. There was no significant evidence in either group for habituation to the open-field task.

\section{Passive Avoidance}

There were no differences between the groups in latency to leave the anterior chamber on either the training or the test days. A comparison of difference scores for Day 1 and Day 2 was significant, however, with Group AA animals having larger difference latencies $(U=6, p<.01)$. This difference was also reflected in the fact that all Group AA rats but only 5 of the 8 Group NA rats remained in the anterior chamber for the full $3 \mathrm{~min}$ of the test.

\section{Eight-Arm Radial Maze}

Performance on the eight-arm radial maze was not significantly different between the groups, although more Group AA rats achieved criterion on the task than did Group NA animals (71\% for Group AA, 25\% for Group NA).

Table 2

Average Daily Fluid Intake (ml/100 g Body Weight) and Standard Errors by Week of Exposure

\begin{tabular}{lccccccccc}
\hline & \multicolumn{10}{c}{ Week } \\
\cline { 2 - 9 } Group & 1 & 2 & 3 & 4 & 5 & 6 & 7 & 8 \\
\hline NA & $25.4 \pm 1.5$ & $25.0 \pm 1.6$ & $22.0 \pm 1.3$ & $19.0 \pm 0.7$ & $16.3 \pm 0.6$ & $14.8 \pm 0.5$ & $13.5 \pm 0.4$ & $12.7 \pm 0.3$ & $11.5 \pm 0.3$ \\
AA & $19.7 \pm 2.2$ & $25.6 \pm 1.4$ & $21.9 \pm 1.0$ & $20.5 \pm 1.8$ & $15.2 \pm 1.2$ & $19.0 \pm 3.7$ & $12.2 \pm 0.8$ & $13.6 \pm 0.7$ & $12.8 \pm 1.2$ \\
\hline
\end{tabular}




\section{DISCUSSION}

In agreement with Thorne et al. (1986), the highest concentrations of aluminum were found in the hippocampal samples, followed by the neocortical and rest-of-brain samples. The hippocampus of developing animals has been reported to have a selective affinity for concentrating metals, such as zinc and lead, which can lead to symptoms of hippocampal dysfunction (see, e.g., Petit, 1984). Hippocampal dysfunction results in deficits on tasks such as passive avoidance, one-way active avoidance, DRL and extinction, and discrimination reversal (Horel, 1978; Isaacson, 1974). In fact, Thome et al. found that high hippocampal aluminum was associated with poor performnce on a PA learning task. By contrast, the present study found no evidence for learning and memory deficits, despite a concentrating of aluminum in the hippocampus of both control and experimental rats. The lack of effect of aluminum on cognitive function in the present study suggests that the age of the animal might have been an important factor in the correlations observed by Thorne et al. That is, the older rats in the Thorne et al. study may have been more susceptible to the toxic effects of aluminum than were the younger animals in the present study, or younger rats may simply incorporate less aluminum because of more efficient kidneys.

Despite the greater period of aluminum administration in the present study, in comparison with that of earlier experiments, there were no between-groups differences in aluminum incorporation for any brain area. It is worth noting, however, that the Group AA rats had larger medians than Group NA rats for each brain area examined. It is probable that the extreme variability in each group, coupled with the relatively small samples, prevented significant results from being obtained.

Unlike animals in previous studies (Commissaris et al., 1982; Thorne et al., 1986), animals exposed to aluminum hydroxide gel in the present study were consistently lighter than control rats. The age of the animals at the time of administration is undoubtedly a factor in this difference, since the present study employed weanling rats and the others employed adult rats. Another possible explanation for the difference is that the route of administration was different. In both of the previous studies, rats were exposed to an aluminum compound admixed with their feed. In the present experiment, weanling rats apparently initially rejected fluid containing the aluminum hydroxide gel. The initial rejection resulted in a weight loss from which Group AA rats never recovered, despite comparable fluid intake on a body weight basis for the remainder of the study.

It is unlikely that the difference in body weight was a direct function of the aluminum. Rather, it is more likely that the young animals found the taste of the aluminum gel initially so aversive that they drank less of the water containing it. In support of this suggestion, comparisons of fluid intake converted to $\mathrm{ml} / 100 \mathrm{~g}$ body weight revealed that Group AA rats drank less of the fluid during the first week of exposure. However, it should also be noted that rest-of-brain aluminum was significantly and negatively correlated with body weight at 6 of 10 measurement periods; that is, the greater the amount of aluminum in the rest-of-brain sections, the lower the body weight. This might suggest a direct effect of aluminum in parts of the brain other than neocortex and hippocampus on body weight.

In agreement with Thorne et al. (1986), aluminum ingestion did not result in increased mouse killing. However, in disagreement with previous research (Bowdler et al., 1979; Commissaris et al., 1982), there were no between-groups differences in activity in the open field. In a few cases, aluminum levels were positively related to horizontal activity but negatively related to rearing. The increased horizontal activity as a function of BAC is in agreement with the increased activity noted by Bowdler et al. in rats intubated with aluminum, but is in opposition to the negative correlations between BAC and horizontal activity reported by Thorne et al.

In contrast to previous studies (Commissaris et al., 1982; Hinz \& Dufort, 1983; King et al., 1975; Thorne et al., 1986) there was no evidence for a deleterious effect of aluminum ingestion (as accomplished in the present study) on cognitive behavior. In fact, by some criteria, Group AA rats actually performed better than Group NA rats. Specifically, Group AA animals had larger difference latencies on the PA task and $71 \%$ achieved criterion on the maze, as did only $25 \%$ of Group NA rats. Although we are hesitant to claim that aluminum ingestion in weanling rats enhances cognitive performance, we feel it is quite likely that it does not disrupt it. The present study suggests that age of administration is a factor in aluminum toxicity studies and is in agreement with reports indicating that developing animals are more resistant to the neurotoxic effects of aluminum (Petit et al., 1985; Wisniewski et al., 1980).

\section{REFERENCES}

Bowdler, N. C., Beasley, D. S., Fritze, E. C., Goulette, A. M., Hatton, J. D., Hession, J., Ostman, D. L., Rugg, D. J., \& SCHMitTdiel, C. J. (1979). Behavioral effects of aluminum ingestion on animal and human subjects. Pharmacology Biochemistry \& Behavior, 10, 505-512.

Commissaris, R. L., Cordon, J. J., Sprague, S., Keiser, J., Mayor, G. H., \&ECH, R. H. (1982). Behavioral changes in rats after chronic aluminum and parathyroid hormone administration. Neurobehavioral Toxicology \& Teratology, 4, 403-410.

Crapper, D. R., \& Dalton, A. J. (1973). Alterations in short-term retention, conditioned avoidance response acquisition and motivation following aluminum induced neurofibrillary degeneration. Physiology \& Behavior, 10, 925-933.

Crapper, D. R., Krishnan, S. S., \& Dalton, A. J. (1973). Brain aluminum distribution in Alzheimer's disease and experimental neurofibrillary degeneration. Science, 180, 511-513.

Crapper, D. R., Krishnan, S. S., \& QuTtKat, S. (1976). Aluminum, neurofibrillary degeneration and Alzheimer's disease. Brain, 99, 67-80.

Hinz, L. D., \& DuforT, R. H. (1983, March). The effect of aluminum on memory of the rat. Paper presented at the meeting of the Southeastern Psychological Association, Atlanta, GA.

Horel, J. A. (1978). The neuroanatomy of amnesia. Brain, 101, 403-445.

IsaAcson, R. L. (1974). The limbic system. New York: Plenum Press. KIng, G. A., DeBoni, U., \& Crapper, D. R. (1975). Effect of aluminum upon conditioned avoidance response acquisition in the absence of neurofibrillary degeneration. Pharmacology Biochemistry \& Behavior, 3, 1003-1009.

Olton, D. S., \& SAMUelson, R. J. (1976). Remembrance of places passed: Spatial memory in rats. Journal of Experimental Psychology: Animal Behavior Processes, 2, 97-116.

PETIT, T. L. (1984). Lead-zinc interactions in the central nervous system, with particular reference to the hippocampus. In C. J. Frederickson, G. A. Howell, \& E. J. Kasarskis (Eds.), The neurobiology of zinc, Part B: Deficiency, toxicity, and pathology. New York: Alan R. Liss.

Petit, T. L., Biederman, G. B., Jonas, P., \& LeBoutillier, J. C. (1985). Neurobehavioral development following aluminum administration in infant rabbits. Experimental Neurology, 88, 640-651.

Petit, T. L., Biederman, G. B., \& McMullen, P. A. (1980). Neurofibrillary degeneration, dendritic dying back, and learning-memory deficits after aluminum administration: Implications for brain aging. Experimental Neurology, 67, 152-162.

Thorne, B. M., Donohoe, T., Lin, K.-N., Lyon, S., Medeiros, D. M., \& WeAVER, M. L. (1986). Aluminum ingestion and behavior in the Long-Evans rat. Physiology \& Behavior, 36, 63-67.

Wisniewski, H. M., Sturman, J. A., \& Shek, J. W. (1980). Aluminum chloride induced neurofibrillary changes in the developing rabbit: A chronic animal model. Annals of Neurology, 8, 479-490.

(Manuscript received for publication October 27, 1986.) 